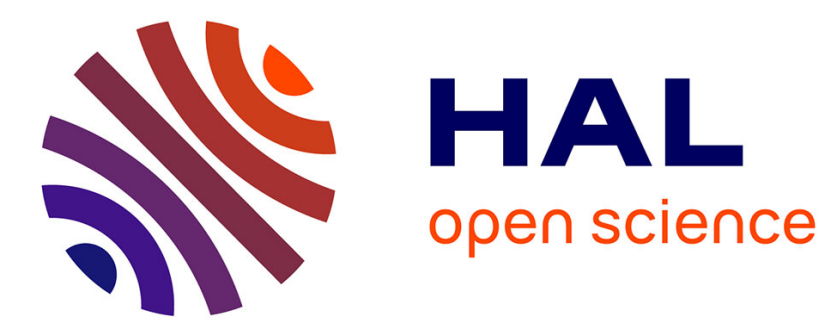

\title{
Plate-like and shell-like inclusions with high rigidity
}

Anne-Laure Bessoud, Françoise Krasucki, Michele Serpilli

\section{To cite this version:}

Anne-Laure Bessoud, Françoise Krasucki, Michele Serpilli. Plate-like and shell-like inclusions with high rigidity. Comptes Rendus. Mathématique, 2008, 346 (11-12), pp.697 - 702. 10.1016/j.crma.2008.03.002 . hal-01829179

\section{HAL Id: hal-01829179 https://hal.science/hal-01829179}

Submitted on 3 Jul 2018

HAL is a multi-disciplinary open access archive for the deposit and dissemination of scientific research documents, whether they are published or not. The documents may come from teaching and research institutions in France or abroad, or from public or private research centers.
L'archive ouverte pluridisciplinaire HAL, est destinée au dépôt et à la diffusion de documents scientifiques de niveau recherche, publiés ou non, émanant des établissements d'enseignement et de recherche français ou étrangers, des laboratoires publics ou privés. 


\title{
Plate-like and shell-like inclusions with high rigidity
}

\author{
Anne-Laure Bessoud ${ }^{\mathrm{a}, \mathrm{b}}$ Françoise Krasucki ${ }^{\mathrm{a}}$, Michele Serpilli ${ }^{\mathrm{b}}$,

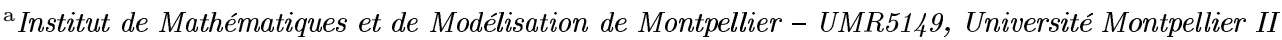 \\ CC 051 Place Eugène Bataillon, 34095 Montpellier cedex 5, France \\ ${ }^{\mathrm{b}}$ Laboratoire de Mécanique et Genie Civil - UMR 5508, Université Montpellier II \\ CC 048 Place Eugène Bataillon, 34095 Montpellier cedex 5, France \\ Received $* * * * *$; accepted after revision +++++ \\ Presented by ččččč
}

\begin{abstract}
We study the problem of an elastic inclusion with high rigidity in a 3D domain. First we consider an inclusion with a plate-like geometry and then in the more general framework of curvilinear coordinates, an inclusion with a shell-like geometry. We compare our formal models to those obtained by Chapelle-Ferent and by Bessoud et al.. To cite this article: A.L. Bessoud, F. Krasucki, M. Serpilli, C. R. Acad. Sci. Paris
\end{abstract}

Résumé

Inclusions élastiques de grande rigidité de type plaque ou coque On étudie le problème d'une inclusion élastique de grande rigidité dans un domaine 3D. Cette inclusion est d'abord vue comme un domaine géométrique de type plaque, puis plus généralement comme un domaine géométrique de type coque. On compare les modèles obtenus formellement à ceux de Chapelle-Ferent et de Bessoud et al.. Pour citer cet article : A.L. Bessoud, F. Krasucki, M. Serpilli, C. R. Acad. Sci. Paris

\section{Introduction}

After the pioneering works of Pham Huy-Sanchez [1], Brezis et al [2] and Caillerie [3], the thin inclusion of a third material between two other ones when the rigidity properties of the inclusion are highly contrasted with respect to those of the surrounding material has been deeply investigated. More recently, Chapelle-Ferent [4] in order to justify some methods used in FEM approximation have studied the asymptotic behavior of a shell-like inclusion of $\frac{1}{\varepsilon^{p}}$-rigidity $(p=1$ or $p=3)$ in a 3D domain. In a slightly different geometrical and mechanical context, Bessoud et al. [5] have studied the behavior of a $\varepsilon$-thin $3 \mathrm{D}$ layer of

Email addresses: bessoud@lmgc.univ-montp2.fr (Anne-Laure Bessoud), krasucki@math.univ-montp2.fr (Françoise Krasucki), serpilli@lmgc.univ-montp2.fr (Michele Serpilli). 
$\frac{1}{\varepsilon}$-rigidity. More precisely, they assume that the thin layer can be written as $\left.\omega \times\right]-\varepsilon, \varepsilon[$ where $\omega$ is a projectable $2 \mathrm{D}$ surface, and that all the materials are linearly elastic anisotropic. Then the limit problem is a Ventcel-type transmission problem between two 3D linearly elastic anisotropic bodies. When $\omega$ is planar and in the isotropic case, the associated surface energy term can be interpreted as the membranal energy of a Kirchhoff-Love plate.

Here two situations are considered using a two fields approach analogous to [4]. In section 2 we study the same geometrical situation as in [5] when the material in the thin layer is isotropic and with a $\frac{1}{\varepsilon^{p}}$-rigidity $(p=1$ or $p=3)$. When $p=3$, in the formal limit problem the associated surface energy corresponds to a flexural energy for a Kirchhoff-Love plate. When $p=1$, the associated surface energy corresponds to the membranal energy of [5].

In section 3 we study a new situation where the shell-like thin layer is obtained by the translation in the normal direction of a general 2D surface, see (2). Using a system of curvilinear coordinates we deduce the formal limit problem for the two cases $p=1$ and $p=3$. In this way we obtain the same limit problems as in [4], also if the kinematical assumptions for the physical problem are not the same. Indeed in [4] the authors a priori assume a shell-like energy in the thin layer. As in [4] one must stress that the well-posedness of the limit problems is essentially linked to the well-posedness of the shell models [6],[7]. $[8]$.

\section{Plate-like inclusion: asymptotic behavior}

In the three-dimensional Euclidean space $\mathcal{E}^{3}$ referred to the Cartesian coordinate frame $\left(O ; \mathbf{e}_{1}, \mathbf{e}_{2}, \mathbf{e}_{3}\right)$, let $\Omega^{+}$and $\Omega^{-}$be two disjoint open domains with smooth boundaries $\partial \Omega^{+}$and $\partial \Omega^{-}$. Let $\omega=\left\{\partial \Omega^{+} \cap \partial \Omega^{-}\right\}^{\circ}$, which is assumed to be a domain in $\mathbb{R}^{2}$ having a positive two-dimensional measure and let $y=\left(y_{\alpha}\right)$ denote a generic point of $\bar{\omega}$. We insert the intermediate layer moving $\Omega^{+}$, (resp. $\Omega^{-}$) in the $\mathbf{e}_{3}$ (resp. $\left.-\mathbf{e}_{3}\right)$ direction of an amount equal to $\varepsilon>0$, a small dimensionless real parameter. Then let $\Omega^{ \pm, \varepsilon}:=\left\{x^{\varepsilon}:=\right.$ $\left.\left.x \pm \varepsilon \mathbf{e}_{3} ; x \in \Omega^{ \pm}\right\}, \Omega^{m, \varepsilon}:=\omega \times\right]-\varepsilon, \varepsilon\left[\right.$, and $\Omega^{\varepsilon}:=\Omega^{-, \varepsilon} \cup \Omega^{+, \varepsilon} \cup \Omega^{m, \varepsilon}$. The structure is clamped on $\Gamma_{0} \subset\left(\partial \Omega^{\varepsilon} \backslash \Gamma^{m, \varepsilon}\right)$ and $\left.\Gamma^{m, \varepsilon}:=\partial \omega \times\right]-\varepsilon, \varepsilon\left[\right.$ is traction free. We suppose that the materials occupying $\Omega^{\varepsilon}$ are linearly elastic and isotropic. Let

$$
V^{\varepsilon}=\left\{(\boldsymbol{V}, \boldsymbol{v}) \in H^{1}\left(\Omega^{\varepsilon} ; \mathbb{R}^{3}\right) \times H^{1}\left(\Omega^{m, \varepsilon} ; \mathbb{R}^{3}\right) ; \boldsymbol{V}_{\mid \Omega^{m, \varepsilon}}=\boldsymbol{v} ; \boldsymbol{V}_{\mid \Gamma_{0}}=0\right\}
$$

The physical variational problem defined over the variable domain $\Omega^{\varepsilon}$ can be written as

$$
\left\{\begin{array}{l}
\text { Find }\left(\boldsymbol{U}^{\varepsilon}, \boldsymbol{u}^{\varepsilon}\right) \in V^{\varepsilon} \text { such that for all }(\boldsymbol{V}, \boldsymbol{v}) \in V^{\varepsilon} \\
A^{-, \varepsilon}\left(\boldsymbol{U}^{\varepsilon}, \boldsymbol{V}^{\varepsilon}\right)+A^{+, \varepsilon}\left(\boldsymbol{U}^{\varepsilon}, \boldsymbol{V}^{\varepsilon}\right)+A^{m, \varepsilon}\left(\boldsymbol{u}^{\varepsilon}, \boldsymbol{v}^{\varepsilon}\right)=L(\boldsymbol{V}),
\end{array}\right.
$$

where

$$
\begin{aligned}
A^{ \pm, \varepsilon}\left(\boldsymbol{U}^{\varepsilon}, \boldsymbol{V}^{\varepsilon}\right) & =\int_{\Omega^{ \pm, \varepsilon}}\left(\lambda^{ \pm, \varepsilon} e_{p p}^{\varepsilon}\left(\boldsymbol{U}^{\varepsilon}\right) e_{q q}^{\varepsilon}\left(\boldsymbol{V}^{\varepsilon}\right)+2 \mu^{ \pm, \varepsilon} e_{i j}^{\varepsilon}\left(\boldsymbol{U}^{\varepsilon}\right) e_{i j}^{\varepsilon}\left(\boldsymbol{V}^{\varepsilon}\right)\right) d x^{\varepsilon} \\
A^{m, \varepsilon}\left(\boldsymbol{u}^{\varepsilon}, \boldsymbol{v}^{\varepsilon}\right) & =\int_{\Omega^{m, \varepsilon}}\left(\lambda^{m, \varepsilon} e_{p p}^{\varepsilon}\left(\boldsymbol{u}^{\varepsilon}\right) e_{q q}^{\varepsilon}\left(\boldsymbol{v}^{\varepsilon}\right)+2 \mu^{m, \varepsilon} e_{i j}^{\varepsilon}\left(\boldsymbol{u}^{\varepsilon}\right) e_{i j}^{\varepsilon}\left(\boldsymbol{v}^{\varepsilon}\right)\right) d x^{\varepsilon}
\end{aligned}
$$

The functional $L(\cdot)$ is the linear application associated with the applied forces.

Let us suppose that the Lamé's constants of the isotropic materials satisfy

$$
\lambda^{ \pm, \varepsilon}=\lambda^{ \pm}, \mu^{ \pm, \varepsilon}=\mu^{ \pm}, \lambda^{m, \varepsilon}=\frac{1}{\varepsilon^{p}} \lambda^{m}, \mu^{m, \varepsilon}=\frac{1}{\varepsilon^{p}} \mu^{m}
$$

where $p \in\{1,3\}$, and $\lambda^{ \pm}, \lambda^{m}$ (resp. $\mu^{ \pm}$, and $\mu^{m}$ ) are of the same order of magnitude. 
In order to study the asymptotic behavior of the solution of the physical problem (1) when $\varepsilon$ tends to zero, we apply the usual change of variable (see [9]), which transforms the problem posed on a $\varepsilon$ dependent domain into a problem over the fixed domain $\Omega:=\Omega^{ \pm, 1} \cup \Omega^{m}$, where $\left.\Omega^{m}:=\omega \times\right]-1,1[$ is the rescaled domain of $\Omega^{m, \varepsilon}$. The rescaled problem assumes the following form, with $V=\{(\boldsymbol{V}, \boldsymbol{v}) \in$ $\left.H^{1}\left(\Omega ; \mathbb{R}^{3}\right) \times H^{1}\left(\Omega^{m} ; \mathbb{R}^{3}\right) ; \boldsymbol{V}_{\mid \Omega^{m}}=\boldsymbol{v} ; \boldsymbol{V}_{\mid \Gamma_{0}}=0\right\}:$

$$
\left\{\begin{array}{l}
\text { Find }(\boldsymbol{U}(\varepsilon), \boldsymbol{u}(\varepsilon)) \in V \text { such that for all }(\boldsymbol{V}, \boldsymbol{v}) \in V \\
A^{-}(\boldsymbol{U}(\varepsilon), \boldsymbol{V})+A^{+}(\boldsymbol{U}(\varepsilon), \boldsymbol{V})+\frac{1}{\varepsilon^{p-1}} A_{\alpha \beta}^{m}(\boldsymbol{u}(\varepsilon), \boldsymbol{v})+\frac{1}{\varepsilon^{p}} A_{\alpha 3}^{m}(\boldsymbol{u}(\varepsilon), \boldsymbol{v})+\frac{1}{\varepsilon^{p+1}} A_{33}^{m}(\boldsymbol{u}(\varepsilon), \boldsymbol{v})=L(\boldsymbol{V})
\end{array}\right.
$$

where $A^{ \pm}(\cdot, \cdot)$ is the natural bilinear form on $\Omega^{ \pm}$, and the bilinear forms $A_{i j}^{m}(\cdot, \cdot)$ are defined as follows:

$$
\begin{gathered}
A_{\alpha \beta}^{m}(\boldsymbol{u}, \boldsymbol{v})=\int_{\Omega^{m}}\left(\lambda^{m} e_{\sigma \sigma}(\boldsymbol{u}) e_{\tau \tau}(\boldsymbol{v})+2 \mu^{m} e_{\alpha \beta}(\boldsymbol{u}) e_{\alpha \beta}(\boldsymbol{v})+\frac{\mu^{m}}{2} \partial_{\alpha} u_{3} \partial_{\alpha} v_{3}\right) d x, \\
A_{\alpha 3}^{m}(\boldsymbol{u}, \boldsymbol{v})=\int_{\Omega^{m}}\left(\frac{\mu^{m}}{2}\left(\partial_{\alpha} u_{3} \partial_{3} v_{\alpha}+\partial_{3} u_{\alpha} \partial_{\alpha} v_{3}\right)+\lambda^{m}\left(e_{\sigma \sigma}(\boldsymbol{u}) e_{33}(\boldsymbol{v})+e_{33}(\boldsymbol{u}) e_{\sigma \sigma}(\boldsymbol{v})\right)\right) d x, \\
A_{33}^{m}(\boldsymbol{u}, \boldsymbol{v})=\int_{\Omega^{m}}\left(\left(\lambda^{m}+2 \mu^{m}\right) e_{33}(\boldsymbol{u}) e_{33}(\boldsymbol{v})+\frac{\mu^{m}}{2} \partial_{3} u_{\alpha} \partial_{3} v_{\alpha}\right) d x .
\end{gathered}
$$

We look for the following formal a priori asymptotic expansion of the solution $(\boldsymbol{U}(\varepsilon), \boldsymbol{u}(\varepsilon))$ :

$$
\boldsymbol{U}(\varepsilon)=\boldsymbol{U}^{0}+\varepsilon \boldsymbol{U}^{1}+\varepsilon^{2} \boldsymbol{U}^{2}+\ldots, \boldsymbol{u}(\varepsilon)=\boldsymbol{u}^{0}+\varepsilon \boldsymbol{u}^{1}+\varepsilon^{2} \boldsymbol{u}^{2}+\ldots .
$$

The leading terms $\left(\boldsymbol{U}^{0}, \boldsymbol{u}^{0}\right)$ of the asymptotic expansion satisfy the limit problems:

(i) $p=1$ :

$$
\left\{\begin{array}{l}
\text { Find }\left(\boldsymbol{U}^{0}, \boldsymbol{u}^{0}\right) \in V_{M} \text { such that for all }(\boldsymbol{V}, \boldsymbol{v}) \in V_{M} \\
A^{-}\left(\boldsymbol{U}^{0}, \boldsymbol{V}\right)+A^{+}\left(\boldsymbol{U}^{0}, \boldsymbol{V}\right)+A_{M}^{m}\left(\boldsymbol{u}^{0}, \boldsymbol{v}\right)=L(\boldsymbol{V})
\end{array}\right.
$$

where

$$
\begin{gathered}
V_{M}=\left\{(\boldsymbol{V}, \boldsymbol{v}) \in H^{1}\left(\Omega ; \mathbb{R}^{3}\right) \times H^{1}\left(\omega ; \mathbb{R}^{3}\right) ; V_{\mid \omega}=\boldsymbol{v}, \boldsymbol{V}_{\mid \Gamma_{0}}=0\right\}, \\
A_{M}^{m}(\boldsymbol{u}, \boldsymbol{v})=\int_{\omega}\left(\frac{4 \lambda^{m} \mu^{m}}{\lambda^{m}+2 \mu^{m}} e_{\sigma \sigma}(\boldsymbol{u}) e_{\tau \tau}(\boldsymbol{v})+4 \mu^{m} e_{\alpha \beta}(\boldsymbol{u}) e_{\alpha \beta}(\boldsymbol{v})\right) d y
\end{gathered}
$$

is the bilinear form associated with the membrane behavior of the plate.

(ii) $p=3$ :

$$
\left\{\begin{array}{l}
\text { Find }\left(\boldsymbol{U}^{0}, \boldsymbol{u}^{0}\right) \in V_{F} \text { such that for all }(\boldsymbol{V}, \boldsymbol{v}) \in V_{F} \\
A^{-}\left(\boldsymbol{U}^{0}, \boldsymbol{V}\right)+A^{+}\left(\boldsymbol{U}^{0}, \boldsymbol{V}\right)+A_{F}^{m}\left(\boldsymbol{u}^{0}, \boldsymbol{v}\right)=L(\boldsymbol{V})
\end{array}\right.
$$

where

$$
\begin{gathered}
V_{F}=\left\{(\boldsymbol{V}, \boldsymbol{v}) \in H^{1}\left(\Omega ; \mathbb{R}^{3}\right) \times H^{2}\left(\omega ; \mathbb{R}^{3}\right) ; \boldsymbol{V}_{\mid \omega}=\boldsymbol{v}, \boldsymbol{V}_{\mid \Gamma_{0}}=0, e_{\alpha \beta}(\boldsymbol{v})=0\right\}, \\
A_{F}^{m}(\boldsymbol{u}, \boldsymbol{v})=\frac{1}{3} \int_{\omega}\left(\frac{4 \lambda^{m} \mu^{m}}{\lambda^{m}+2 \mu^{m}} \Delta_{\tau} u_{3} \Delta_{\tau} v_{3}+4 \mu^{m} \partial_{\alpha \beta} u_{3} \partial_{\alpha \beta} v_{3}\right) d y
\end{gathered}
$$

is the bilinear form associated with the flexural behavior of the plate. $\Delta_{\tau}$ denotes the two-dimensional Laplacian operator in $\omega$.

Remarks: 1 . This problem can be studied in the case of anisotropy of materials and with less restrictive assumptions relative to the geometry of the central layer $\Omega^{m, \varepsilon}$.

2. In the case $p=1$, the convergence is studied with a slightly different formulation in [5]. 


\section{Shell-like inclusion: asymptotic behavior}

Let $\boldsymbol{\theta} \in \mathcal{C}^{2}\left(\bar{\omega} ; \mathbb{R}^{3}\right)$ be an injective mapping such that the vectors $\boldsymbol{a}_{\alpha}(y):=\partial_{\alpha} \boldsymbol{\theta}(y)$ form the covariant basis of the tangent plane to the surface $S:=\boldsymbol{\theta}(\bar{\omega})$ at the point $\boldsymbol{\theta}(y)$; the two vectors $\boldsymbol{a}^{\alpha}(y)$ of the tangent plane, defined by the relations $\boldsymbol{a}^{\alpha}(y) \cdot \boldsymbol{a}_{\beta}(y)=\delta_{\beta}^{\alpha}$, form its contravariant basis. Also let $\boldsymbol{a}_{3}(y)=\boldsymbol{a}^{3}(y):=$ $\frac{\boldsymbol{a}_{1}(y) \wedge \boldsymbol{a}_{2}(y)}{\left|\boldsymbol{a}_{1}(y) \wedge \boldsymbol{a}_{2}(y)\right|}$ be the unit normal vector to $S$. The covariant and contravariant components $a_{\alpha \beta}$ and $a^{\alpha \beta}$ of the first fundamental form, the covariant and mixed components $b_{\alpha \beta}$ and $b_{\alpha}^{\beta}$ of the second fundamental form, and the Christoffel symbols $\Gamma_{\alpha \beta}^{\sigma}$ of the surface $S$ are then defined by letting:

$$
a_{\alpha \beta}:=\boldsymbol{a}_{\alpha} \cdot \boldsymbol{a}_{\beta}, \quad a^{\alpha \beta}:=\boldsymbol{a}^{\alpha} \cdot \boldsymbol{a}^{\beta}, \quad b_{\alpha \beta}:=\boldsymbol{a}^{3} \cdot \partial_{\beta} \boldsymbol{a}_{\alpha}, b_{\alpha}^{\beta}:=a^{\beta \sigma} b_{\alpha \sigma}, \quad \Gamma_{\alpha \beta}^{\sigma}:=\boldsymbol{a}^{\sigma} \cdot \partial_{\beta} \boldsymbol{a}_{\alpha} .
$$

Let $\left.\Omega^{m, \varepsilon}:=\omega \times\right]-\varepsilon, \varepsilon\left[\right.$ be defined as previously, with $\Gamma^{ \pm, \varepsilon}:=\omega \times\{ \pm \varepsilon\}$. Let $x^{\varepsilon}$ denote the generic point in the set $\bar{\Omega}^{m, \varepsilon}$, with $x_{\alpha}^{\varepsilon}=y_{\alpha}$. We consider a shell-like domain with middle surface $S=\boldsymbol{\theta}(\bar{\omega})$ and thickness $2 \varepsilon>0$, whose reference configuration is the image $\boldsymbol{\Theta}^{m, \varepsilon}\left(\bar{\Omega}^{m, \varepsilon}\right) \subset \mathbb{R}^{3}$ of the set $\bar{\Omega}^{m, \varepsilon}$ through the mapping $\Theta^{m, \varepsilon}: \bar{\Omega}^{m, \varepsilon} \rightarrow \mathbb{R}^{3}$ given by

$$
\boldsymbol{\Theta}^{m, \varepsilon}\left(x^{\varepsilon}\right):=\boldsymbol{\theta}(y)+x_{3}^{\varepsilon} \boldsymbol{a}_{3}(y), \text { for all } x^{\varepsilon}=\left(y, x_{3}^{\varepsilon}\right)=\left(y_{1}, y_{2}, x_{3}^{\varepsilon}\right) \in \bar{\Omega}^{m, \varepsilon} .
$$

Moreover, we suppose that there exists an immersion $\Theta^{\varepsilon}: \bar{\Omega}^{\varepsilon} \rightarrow \mathbb{R}^{3}$ defined as follows:

$$
\boldsymbol{\Theta}^{\varepsilon}:=\left\{\begin{array}{l}
\boldsymbol{\Theta}^{ \pm, \varepsilon} \text { on } \bar{\Omega}^{ \pm, \varepsilon} \\
\boldsymbol{\Theta}^{m, \varepsilon} \text { on } \bar{\Omega}^{m, \varepsilon}
\end{array}, \Theta^{ \pm, \varepsilon}\left(\Gamma^{ \pm, \varepsilon}\right)=\boldsymbol{\Theta}^{m, \varepsilon}\left(\Gamma^{ \pm, \varepsilon}\right),\right.
$$

with $\Theta^{ \pm, \varepsilon}: \bar{\Omega}^{ \pm, \varepsilon} \rightarrow \mathbb{R}^{3}$ immersions over $\bar{\Omega}^{ \pm, \varepsilon}$ defining the curvilinear coordinates on $\bar{\Omega}^{ \pm, \varepsilon}$.

The physical variational problem in these curvilinear coordinates on the variable domain $\Omega^{\varepsilon}$ is

$$
\left\{\begin{array}{l}
\text { Find }\left(\boldsymbol{U}^{\varepsilon}, \boldsymbol{u}^{\varepsilon}\right) \in V^{\varepsilon} \text { such that for all }\left(\mathbf{V}^{\varepsilon}, \boldsymbol{v}^{\varepsilon}\right) \in V^{\varepsilon} \\
A^{-, \varepsilon}\left(\boldsymbol{U}^{\varepsilon}, \boldsymbol{V}^{\varepsilon}\right)+A^{+, \varepsilon}\left(\boldsymbol{U}^{\varepsilon}, \boldsymbol{V}^{\varepsilon}\right)+A^{m, \varepsilon}\left(\boldsymbol{u}^{\varepsilon}, \boldsymbol{v}^{\varepsilon}\right)=L\left(\boldsymbol{V}^{\varepsilon}\right),
\end{array}\right.
$$

where

$$
\begin{aligned}
A^{ \pm, \varepsilon}\left(\boldsymbol{U}^{\varepsilon}, \boldsymbol{V}^{\varepsilon}\right) & :=\int_{\Omega^{ \pm, \varepsilon}} A_{ \pm}^{i j k \ell, \varepsilon} e_{k \ell}^{\varepsilon}\left(\mathbf{U}^{\varepsilon}\right) e_{i j}^{\varepsilon}\left(\mathbf{V}^{\varepsilon}\right) \sqrt{g^{ \pm, \varepsilon}} d x^{\varepsilon}, \\
A^{m, \varepsilon}\left(\boldsymbol{u}^{\varepsilon}, \boldsymbol{v}^{\varepsilon}\right) & :=\int_{\Omega^{m, \varepsilon}} A_{m}^{i j k \ell, \varepsilon} e_{k \ell}^{\varepsilon}\left(\boldsymbol{u}^{\varepsilon}\right) e_{i j}^{\varepsilon}\left(\boldsymbol{v}^{\varepsilon}\right) \sqrt{g^{m, \varepsilon}} d x^{\varepsilon} .
\end{aligned}
$$

Here $A^{i j k \ell, \varepsilon}:=\lambda^{\varepsilon} g^{i j, \varepsilon} g^{k \ell, \varepsilon}+\mu^{\varepsilon}\left(g^{i k, \varepsilon} g^{j \ell, \varepsilon}+g^{i \ell, \varepsilon} g^{j k, \varepsilon}\right)$ are the contravariant components of the elasticity tensor, $g^{\varepsilon}:=\operatorname{det}\left(g_{i j}^{\varepsilon}\right)$, with $\left(g_{i j}^{\varepsilon}\right):=\left(\partial_{i} \Theta^{\varepsilon} \cdot \partial_{j} \boldsymbol{\Theta}^{\varepsilon}\right)$ and the $\left(g^{i j, \varepsilon}\right):=\left(g_{i j}^{\varepsilon}\right)^{-1}$ respectively the covariant and contravariant components of the metric tensor associated with $\Theta^{\varepsilon}$.

As in the previous section, in order to study the asymptotic behavior of the physical problem (3), we apply the usual change of variable, which transforms $\Omega^{\varepsilon}$ into a fixed domain $\Omega$.

Now, the leading terms $\left(\boldsymbol{U}^{0}, \boldsymbol{u}^{0}\right)$ of the asymptotic expansion satisfy the following limit problems:

(i) $p=1$ :

$$
\left\{\begin{array}{l}
\text { Find }\left(\boldsymbol{U}^{0}, \boldsymbol{u}^{0}\right) \in V_{M} \text { such that for all }(\boldsymbol{V}, \boldsymbol{v}) \in V_{M} \\
A^{-}\left(\boldsymbol{U}^{0}, \boldsymbol{V}\right)+A^{+}\left(\boldsymbol{U}^{0}, \boldsymbol{V}\right)+A_{M}^{m}\left(\boldsymbol{u}^{0}, \boldsymbol{v}\right)=L(\boldsymbol{V})
\end{array}\right.
$$

where

$$
\begin{gathered}
V_{M}=\left\{(\boldsymbol{V}, \boldsymbol{v}) \in H^{1}\left(\Omega ; \mathbb{R}^{3}\right) \times H^{1}\left(\omega ; \mathbb{R}^{3}\right) ; \boldsymbol{V}_{\mid \omega}=\boldsymbol{v}, \boldsymbol{V}_{\mid \Gamma_{0}}=0\right\} \\
A_{M}^{m}(\boldsymbol{u}, \boldsymbol{v})=\int_{\omega} a^{\alpha \beta \sigma \tau} \gamma_{\sigma \tau}(\boldsymbol{u}) \gamma_{\alpha \beta}(\boldsymbol{v}) \sqrt{a} d y
\end{gathered}
$$


is the bilinear form associated with the membrane behavior of the shell, $a^{\alpha \beta \sigma \tau}:=\frac{4 \lambda^{m} \mu^{m}}{\lambda^{m}+2 \mu^{m}} a^{\alpha \beta} a^{\sigma \tau}+$ $2 \mu^{m}\left(a^{\alpha \sigma} a^{\beta \tau}+a^{\alpha \tau} a^{\beta \sigma}\right)$ are the contravariant components of the elasticity tensor of the shell and $\gamma_{\alpha \beta}(\boldsymbol{u}):=\frac{1}{2}\left(\partial_{\alpha} u_{\beta}+\partial_{\beta} u_{\alpha}\right)-\Gamma_{\alpha \beta}^{\sigma} u_{\sigma}-b_{\alpha \beta} u_{3}$ are the covariant components of the change of metric tensor.

(ii) $p=3$ :

where

$$
\left\{\begin{array}{l}
\text { Find }\left(\boldsymbol{U}^{0}, \boldsymbol{u}^{0}\right) \in V_{F} \text { such that for all }(\boldsymbol{V}, \boldsymbol{v}) \in V_{F} \\
A^{-}\left(\boldsymbol{U}^{0}, \boldsymbol{V}\right)+A^{+}\left(\boldsymbol{U}^{0}, \boldsymbol{V}\right)+A_{F}^{m}\left(\boldsymbol{u}^{0}, \boldsymbol{v}\right)=L(\boldsymbol{V})
\end{array}\right.
$$

$$
\begin{gathered}
V_{F}=\left\{(\boldsymbol{V}, \boldsymbol{v}) \in H^{1}\left(\Omega ; \mathbb{R}^{3}\right) \times H^{2}\left(\omega ; \mathbb{R}^{3}\right) ; \boldsymbol{V}_{\mid \omega}=\boldsymbol{v}, \boldsymbol{V}_{\mid \Gamma_{0}}=0, \gamma_{\alpha \beta}(\boldsymbol{v})=0 \text { in } \omega\right\}, \\
A_{F}^{m}(\boldsymbol{u}, \boldsymbol{v})=\frac{1}{3} \int_{\omega} a^{\alpha \beta \sigma \tau} \rho_{\sigma \tau}(\boldsymbol{u}) \rho_{\alpha \beta}(\boldsymbol{v}) \sqrt{a} d y
\end{gathered}
$$

is the bilinear form associated with the flexural behavior of the shell and $\rho_{\alpha \beta}(\boldsymbol{u}):=\partial_{\alpha \beta} u_{3}-$ $\Gamma_{\alpha \beta}^{\sigma} \partial_{\sigma} u_{3}-b_{\alpha \beta} u_{3}+b_{\alpha}^{\sigma}\left(\partial_{\beta} u_{\sigma}-\Gamma_{\beta \sigma}^{\tau} u_{\tau}\right)+b_{\beta}^{\tau}\left(\partial_{\alpha} u_{\tau}-\Gamma_{\alpha \tau}^{\sigma} u_{\sigma}\right)+\left(\partial_{\alpha} b_{\beta}^{\tau}+\Gamma_{\alpha \sigma}^{\tau} b_{\beta}^{\sigma}-\Gamma_{\alpha \beta}^{\sigma} b_{\sigma}^{\tau}\right) u_{\tau}$ are the covariant components of the change of curvature tensor.

Remark: As in [4] the well-posedness of the limit problems (i) and (ii) depends both on the Hilbertian character of $V_{M}$ and $V_{F}$, and the coerciveness of the bilinear form $A_{M}^{m}$. For the well-posedness of the flexural shell model see [10], [11] and for the well posedness of the membrane shell model see [12], [13].

\section{References}

[1] H. Pham Huy, E. Sanchez-Palencia, Phénomène de transmission à travers des couches minces de conductivité élevée, J. Math. Anal. Appl. 47 (1974) 284-309.

[2] H.Brezis, L. A. Caffarelli, A. Friedman, Reinforcement problems for elliptic equations and variational inequalities, Ann. Mat. Pura Appl., s. 4, 123 (1980) 219-246.

[3] D. Caillerie, The effect of a thin inclusion of high rigidity in an elastic body, Math. Methods Appl. Sci. 2 (1980) 251-270.

[4] D. Chapelle, A. Ferent, Modeling of the inclusion of a reinforcing sheet within a 3D medium, Math. Models Methods Appl. Sci. 13 (2003) 573-595.

[5] A.L. Bessoud, F. Krasucki, G. Michaille, Multi-materials with strong interface: variational modelings, (submitted).

[6] J. Sanchez-Hubert, E. Sanchez-Palencia, Coques élastiques minces: propriétés asymptotiques, Masson, Paris, 1997.

[7] P.G. Ciarlet, Mathematical Elasticity, vol. III: Theory of shells, Studies in mathematics and its applications, NorthHolland, Amsterdam, 2000.

[8] K. J. Bathe, D. Chapelle, The finite element analysis of shells-fundamentals, Springer, Berlin, 2003.

[9] G. Geymonat, F. Krasucki, S. Lenci, Mathematical analysis of a bonded joint with a soft thin adhesive, Math. Mech. Solids 4 (1999) 201-225.

[10] M. Bernadou, P.G. Ciarlet, Sur l'ellipticité du modèle linéaire de coques de W.T: Koiter, Computing Methods in Applied Scieces and Engineering, R. Glowinski and J.L. Lions Editors, pp. 89-136, Lecture Notes in Economics and Mathematical Systems, 134, Springer-Verlag, Heidelberg, 1976

[11] M. Bernadou, P.G. Ciarlet, B. Miara, Existence theorems for two-dimensional linear shell theories, J. Elasticity, 34, (1994), 111-138.

[12] P.G. Ciarlet, V. Lods, Asymptotic analysis of linearly elastic shells. I. Justification of membrane shell equations, Arch. Rational Mech. Anal. 136 (1996a) 119-161

[13] P.G. Ciarlet, E. Sanchez-Palencia, An existence and uniqueness theorem for the two-dimensional linear membrane shell equations, J. Math. Pures Appl. 75 (1996) 51-67 